\title{
«LA FABRIQUE DES SPORTS NATIONAUX ». ÉTUDIER L'ÉMERGENCE D’UNE ÉLITE DU SPORT SUISSE (1860-1930)
}

\author{
Philippe Vonnard, Gil Mayencourt, Hans-Dieter Gerber
}

De Boeck Supérieur | «Staps »

2019/3 nº 125 | pages 151 à 163

ISSN 0247-106X

ISBN 9782807393394

Article disponible en ligne à l'adresse :

https://www.cairn.info/revue-staps-2019-3-page-151.htm

Distribution électronique Cairn.info pour De Boeck Supérieur.

(C) De Boeck Supérieur. Tous droits réservés pour tous pays.

La reproduction ou représentation de cet article, notamment par photocopie, n'est autorisée que dans les limites des conditions générales d'utilisation du site ou, le cas échéant, des conditions générales de la licence souscrite par votre établissement. Toute autre reproduction ou représentation, en tout ou partie, sous quelque forme et de quelque manière que ce soit, est interdite sauf accord préalable et écrit de l'éditeur, en dehors des cas prévus par la législation en vigueur en France. Il est précisé que son stockage dans une base de données est également interdit. 


\section{« La Fabrique des sports nationaux ॥. Étudier \\ l'émergence d'une élite du sport Suisse (1860-1930)}

\section{"La Fabrique des sports nationaux." Studying the \\ development of the Swiss sport elite (1860-1930)}
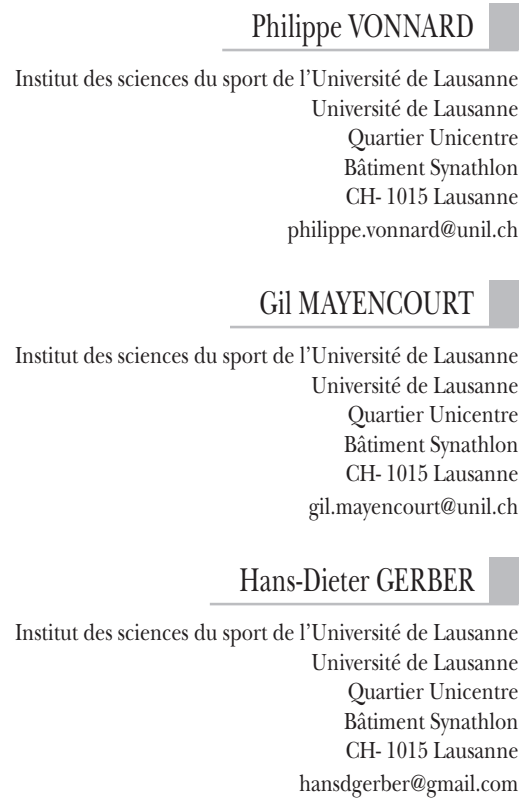

Résumé : En Suisse comme ailleurs, les effets et les résonances des activités sportives touchent à la fois aux sphères économiques, politiques, hygiéniques ou culturelles, et l'institutionnalisation du sport est un processus qui permet de comprendre singulièrement des dynamiques structurantes de notre modernité.

Tenant compte de cette situation et dans le but de comprendre la place importante prise par le sport dans la société suisse, le projet intitulé «La Fabrique des sports nationaux »-financé pour la période 2019-2023 par le Fonds national suisse pour la recherche scientifique (FNS) - propose de se concentrer sur la genèse et les premiers développements des sports en Suisse de 1860 à 1930. Ce processus d'établissement des principaux sports dans la société helvétique suit plus généralement le développement politique, économique et culturel du pays lui-même dont la structure étatique moderne date de 1848. La recherche porte sur le développement de cinq sports (par ordre alphabétique) : le cyclisme, la gymnastique, le football, le tir, et ce que l'on appelle généralement les « Jeux nationaux » constitués par le hornuss et la lutte suisse.

Dans le cadre de cet article, il s'agit de revenir brièvement sur les objectifs de ce projet, puis de développer un aspect central de celui-ci : la recherche extensive engagée autour des élites du sport suisse.

Mots-cĹÉs : Histoire, Sport, Suisse, organisations, élites 
Aвsтract: In Switzerland as elsewhere, the effects and resonances of sporting activities affect the economic, political, health, and cultural spheres, and the institutionalization of sport is a process that makes it possible to understand the structuring dynamics of our modernity in a unique way. Considering those facts and in order to understand the important role played by sport in Swiss society, the "La Fabrique des sports nationaux" project—funded between 2019 and 2023 by the Swiss National Science Foundation (SNSF) - proposes to focus on the origins and early development of sport in Switzerland from 1860 to 1930. This process of establishing the main sports in Swiss society more generally follows the political, economic, and cultural development of the country itself, whose modern state structure dates back to 1848 . The research focuses on the development of five sports: cycling, gymnastics, football, shooting, and what are generally referred to as "national games," consisting of Hornussen and Swiss wrestling.

In this article, we will briefly summarize the main objectives of this project and then develop a central aspect of it: the extensive research on Swiss sports elites.

KEYWORDs: history, sport, Switzerland, organizations, elites

\section{INTRODUCTION}

Les organisateurs de la Fête fédérale de lutte qui s'est tenue à Zoug en août 2019 ont décidé de construire un stade provisoire de 50000 places, ce qui en fait l'un des plus grands de l'histoire de tous les sports. De même, les promoteurs de la prochaine Fête fédérale de gymnastique qui se déroulera à Lausanne en 2025 estiment qu'environ 70000 gymnastes participeront à l'événement - en comparaison, 11000 athlètes ont pris part aux Jeux olympiques de Rio de Janeiro en 2016. En outre, dans chaque agglomération suisse (des grandes cités aux petits villages), il est possible de trouver des sociétés de gymnastiques et/ou des clubs de football. De plus, il est bien connu que les citoyens helvétiques ont tendance à consacrer beaucoup de leur temps aux activités sportives et s'investissent (souvent en tant que bénévole) dans le système sportif (au sein de clubs/associations ou pour l'organisation d'événements). Force est de constater que la pratique et l'associationnisme sportif occupent une place majeure dans la société helvétique.

Si les sports impliquent une économie (des athlètes professionnels au marché mondial du vêtement sportif) et des discussions politiques (autour, par exemple, de la construction des infrastructures), leur impact dépasse largement le domaine sportif en lui-même. Nous pouvons affirmer que les sports - certes à des degrés divers selon les pratiques - jouent un rôle non-négligeable dans la construction de l'identité suisse. En effet, les matchs des équipes nationales (par exemple de football) ou de personnalités sportives (comme le joueur de tennis Roger Federer) offrent aux citoyens suisses la possibilité de se rencontrer (directement ou indirectement grâce à la télévision) et de créer - au travers de la " communauté imaginée " (Anderson, 1996) produite autour des événements - des croyances communes. En outre, comme le souligne Hobsbawm, les sports ont ceci de spécifique que, d'une part, ils concernent une grande majorité de citoyens qui ont la possibilité de comprendre aisément ce qui se déroule, sur les terrains ou dans les stades. D'autre part, les suiveurs ont la possibilité de s'identifier au succès ou à l'échec des icônes sportives (Hobsbawm, 1991, p. 264). C'est pour ces différentes raisons que les sports ont contribué - comme l'iconographie des paysages alpins (Walter, 2004, p. 247) - à la construction et/ou au renforcement de l'identité suisse, et in fine à l'unité de l'État helvétique. Ce point est particulièrement important dans la société suisse en raison de l'existence 
de profonds clivages (culturels, sociaux et religieux) entre les citoyens du pays'.

Pour comprendre cette place importante prise par le sport dans la société suisse, le projet intitulé «La Fabrique des sports nationaux " - financé pour la période 2019-2023 par le Fonds national suisse pour les sciences (FNS) (http://p3. snf.ch/project-182399) - propose de se concentrer sur la genèse et les premiers développements des sports en Suisse de 1860 à 1930. Ce processus d'établissement des principaux sports dans la société helvétique suit plus généralement le développement politique, économique et culturel du pays lui-même, dont la structure étatique moderne date de 1848. La recherche porte sur le développement de cinq sports (par ordre alphabétique) : le cyclisme, la gymnastique, le football, le tir, et ce que l'on appelle généralement les " Jeux nationaux » constitués par le hornuss et la lutte suisse ${ }^{2}$.

Le projet a pour objectif principal de saisir un double processus. D'une part, l'« hybridation " progressive de pratiques étrangères comme le football - bien révélée par la création d'une équipe nationale suisse composée de citoyens suisses, la disparition de l'anglais comme langue parlée au sein d'une grande partie des clubs mais aussi le début d'une subvention nationale donnée par la Confédération à l'Association suisse de football (ASF) dès le début des années 1920. D'autre part, nous souhaitons étudier la "sportivisation » de certaines pratiques, comme la gymnastique - avec notamment la création de compétitions et une démilitarisation progressive de ses contenus. Afin de comprendre ce double processus, il sera nécessaire de se concentrer sur les principaux promoteurs des associations nationales des cinq sports que nous avons sélectionnés au préalable. Trois questions principales nous intéressent particulièrement dans le cadre de ce projet : quelle était la trajectoire sociale de ces personnes? Quelles étaient leurs motivations à investir beaucoup de temps dans le développement de ces activités et enfin quels liens - s'il y en avait - entretenaient-ils entre eux?

Le présent article est divisé en deux parties. Dans la première, nous décrirons l'état de l'histoire du sport en Suisse, une démarche qui permettra de souligner autant l'apport du projet au regard de l'historiographie existante que la nécessité d'étudier la période sélectionnée. Dans la deuxième partie, nous insisterons davantage sur les recherches que nous mènerons sur les élites sportives et indiquerons brièvement quelques éléments sur nos démarches théoriques et empiriques.

\section{FaIRE UN PAS SUPPLÉMENTAIRE dANS L'HistoriographIE dU SPORT EN SUISSE}

Avant le début de ce siècle, l'histoire du sport en Suisse restait peu étudiée et les publications étaient principalement le fruit de journalistes ou d'historiens non professionnels. Quelques (rares) exceptions existaient néanmoins, comme la thèse de doctorat de Louis Burgener consacrée à l'histoire de l'institutionnalisation de l'éducation physique dans la société suisse (1952) - suivie de plusieurs articles publiés dans la Revue suisse militaire ou les quelques livres comme celui écrit par Lutz Eichenberger sur la Commission fédérale de gymnastique (1994) et celui de Geneviève Heller sur le rôle de la gymnastique dans le contrôle de l'enfant à l'école au XIX ${ }^{\mathrm{e}}$ siècle (1988). Notons aussi les réflexions générales

1 Contrairement à l'image internationale pacifique de la Suisse, le pays connaît régulièrement des moments de tensions économiques, politiques ou sociales (voir notamment Jost, 2001 ; Villiger, 2017). Cependant, si ces tensions créent quelques difficultés dans la société suisse, il semble que celles-ci constituent moins un danger pour l'existence même de l'État par rapport à ce qu'il se passe par exemple en Belgique (pays également marqué par de profondes divisions culturelles, linguistiques et religieuses).

2 Le ski, qui peut également être considéré comme l'un des sports principaux en Suisse, n'a pas été sélectionné dans le projet. Ce choix s'explique par le fait que Sébastien Cala (assistant-diplômé à l'UNIL) étudie déjà ce sport dans le cadre d'une thèse. Comme indiqué en fin d'article, Sébastien Cala fait partie intégrante de l'équipe du projet. 
de Fritz Pieth sur le développement du sport dans le pays (1979).

Si ces travaux ont fourni des bases intéressantes (notamment en ce qui concerne la chronologie et les acteurs impliqués dans le domaine sportif), le nombre d'études consacrées à l'histoire du sport - ou plutôt des sports - en Suisse reste faible par rapport aux pays voisins (par exemple : Holt, 1981 ; Arnaud, 1987, Eisenberg, 1999 ; Pfister, 2003). Un changement a néanmoins commencé à s'opérer au début des années 2000 grâce au travail d'une nouvelle génération de chercheurs. À l'heure actuelle, le sport le plus étudié est le football. Des recherches ont notamment été menées sur l'implantation et les premiers développements du jeu à la fin du XIX ${ }^{\mathrm{e}}$ siècle (Koller, 2017 ; Gogniat, 2018). La période la plus connue est celle de l'entre-deux-guerres, grâce en particulier aux recherches de Fabien Brändle et Christian Koller qui ont étudié de manière approfondie la démocratisation, la commercialisation et la politisation du jeu durant cette période (voir par exemple leur ouvrage de synthèse paru en 2014). Le lien entre le football et la construction de la nation a aussi été mis quelque peu à jour, notamment par l'entremise d'études sur la couverture des rencontres de l'équipe nationale par la presse durant les années 1930 (Quin, 2010 ; Quin \& Vonnard, 2011). Des auteurs ont également travaillé sur la question de la professionnalisation des joueurs et ont pu dégager les différentes phases qu'a connues le processus (Vonnard \& Quin, 2012 ; Berthoud, Quin \& Vonnard, 2016, 2018). Enfin, des recherches menées sur les premières années du FC Bâle (Gerber, 2001) et du Red Star Zurich (Koller, 2012) ont permis d'entamer des réflexions à une échelle plus locale.

Certes, les études sur le football sont les plus développées, toutefois des travaux intéressants ont également été réalisés sur d'autres sports. C'est par exemple le cas pour les sports d'hiver (principalement le ski alpin et le hockey sur glace). Nous pouvons indiquer ici des articles sur l'arrivée du ski (Philipona Romanens,
1999 ; Busset \& Marcacci, 2006 ; Busset, 2016) et du hockey sur glace (Busset, 2001 ; Koller, 2017) dans certaines régions du pays (en particulier la Suisse romande). Des chercheurs ont par ailleurs souligné le lien étroit qui unit sport et tourisme (Tissot, 2000 ; Tissot, Quin \& Vonnard, 2019) et ont notamment montré que les hôteliers ont joué un rôle majeur dans le développement de plusieurs pratiques (comme le ski, la luge et le tennis) dans le pays (Barton, 2008 ; Favre \& Vonnard, 2015 ; Cala, 2019 ; Tissot \& Schneider, 2019). De plus, à la suite des recherches pionnières de Jean-Claude Bussard sur l'établissement progressif du sport en tant que discipline dans le système scolaire (voir notamment sa thèse de doctorat publiée en 2007), c'est la place de la gymnastique à l'école (Czáka, 2017) ou plus généralement du sport à l'université (Quin, 2016) qui ont été étudiés de manière approfondie. À propos du cas de la gymnastique, il faut aussi souligner l'importante contribution d'Ingrid Brühwiler qui a traité des enjeux de l'arrivée de la gymnastique suédoise dans le pays dans le courant du XIXe siècle (par exemple : 2017).

Signalons encore qu'à la suite des premières réflexions conduites par Pierre Lanfranchi à la fin des années 1990 sur le rôle des citoyens suisses dans les premiers développements du football en Europe (1998), des approfondissements ont été apportés sur les périodes de l'entre-deux-guerres (Koller, 2010 ; Quin \& Vonnard, 2015) ou encore sur la place occupée par les dirigeants helvètes au sein de la Fédération internationale de football association (FIFA) (Vonnard \& Quin, 2019). Dans cette optique, nous pouvons mentionner les études sur la participation de la Suisse aux Jeux olympiques d'été de 1936 à Berlin (Favre, 2004) ou, au contraire, la non-participation des athlètes de la Confédération aux Jeux olympiques d'été de 1956 à Melbourne (Tonnerre \& Quin, 2017). Enfin, s'inspirant de recherches conduites autour de la diplomatie culturelle de la Suisse (par exemple : Gillabert, 2013), des réflexions sont en cours sur l'utilisation du 
sport pour la diplomatie politique ou économique suisse (Tonnerre, 2019), un domaine de recherche qui bénéficiera grandement de la thèse que prépare actuellement Quentin Tonnerre à l'Université de Lausanne ${ }^{3}$.

Ce bref, et non exhaustif, état de l'art démontre qu'un processus positif existe autour du développement de l'histoire du sport dans le pays, cet aspect étant appuyé par la création de l'Association suisse d'histoire du sport (ASHS) (Vonnard, 2019) ainsi que par deux ouvrages récemment parus qui fournissent assurément de nouveaux apports au regard des thématiques abordées et du matériel empirique collecté par les auteurs (Busset, Jucker \& Koller, 2019 ; Quin, Vonnard \& Jaccoud, 2019) ${ }^{4}$.

Malgré les avancées constatées ces deux dernières décennies, il reste encore beaucoup à faire pour comprendre l'essor et le développement des sports en Suisse ainsi que leur place dans la société helvétique. Trois remarques principales peuvent être faites sur cet état de l'art. Premièrement, des sports extrêmement populaires, comme le cyclisme, le tir ainsi que le hornuss et la lutte suisse (appelés également " les jeux nationaux ») sont clairement sous-étudiés. Or, dans d'autres pays, des historiens ont montré par exemple à quel point le tir - en raison de ses relations étroites avec l'armée - était important pour la formation d'une unité nationale (Pécout, 1990). Pour le cyclisme, des spécialistes ont également indiqué pour le cas de la France (Gaboriau, 1991 ; Wille, 2003) ou de l'Angleterre (voir en particulier les recherches en cours de Neil Carter), que ce sport était l'un des plus populaires à la fin du XIX ${ }^{e}$ siècle et a été très tôt professionnalisé. En ce qui concerne le hornuss et la lutte, il serait intéressant que des chercheurs suisses s'inspirent des travaux conduits sur les jeux nationaux irlandais et notamment sur leur rôle dans la construction des identités locales et nationales (par exemple : Cronin, Duncan \& Rouse, 2009).
Deuxièmement, nous ne connaissons pas bien l'institutionnalisation des sports suisses au niveau national. Hormis des études préliminaires sur le cas du football (Guggisberg, 2009 ; Quin \& Vonnard, 2019) ou du ski (Quin, 2017), nous ne disposons pas de recherches précises sur les enjeux de la création et du développement de fédérations nationales. Dans le même ordre d'idées, très peu d'études ont été conduites sur les dirigeants sportifs. Nous ne savons donc pas véritablement qui ils étaient et pourquoi ils ont décidé d'investir beaucoup de temps dans le développement du sport.

Troisièmement, la relation entre sport et politique ou entre sport et économie reste peu étudiée. Sur ce point, nous pouvons néanmoins indiquer la monographie de Markus Giuliani sur le système sportif suisse et ses relations avec l'État de 1918 à 1947 (2001). En ce qui concerne l'économie, Laurent Tissot a récemment offert une réflexion générale dans un article qui pourrait aider à développer des recherches dans un avenir proche (2018). Dans le but de répondre à certains de ces manques, en particulier ceux relatifs aux enjeux de l'institutionnalisation des principaux sports, trois axes différents seront explorés au cours des quatre années du projet "La Fabrique des sports nationaux" :

- Nous nous concentrerons sur les enjeux de la création des fédérations nationales des sports sélectionnés. Les questions principales sont : pourquoi et comment ces organisations ont-elles été créées/développées. Quelles étaient leurs fonctions principales ? Et comment celles-ci ont-elles changé au fil du temps ?

- Ensuite, nous identifierons l'élite (membres du comité exécutif) de ces organisations. Les questions principales sont : quel était le profil social des membres de ces élites? Et quels étaient les objectifs de leur fort investissementces acteurs ayant passé beaucoup de temps, la plupart du temps sans gagner d'argent, dans les activités de ces organisations?

3 Sous la direction des Professeurs Patrick Clastres et Janick Marina Schaufelbuehl.

4 Pour des compléments à cet état de l'art, voir Gigase, Jucker \& Koller (2016) et l'introduction des deux livres indiqués. 
- Enfin, nous étudierons les interactions entre ces fédérations. En effet, dans les études précitées, Jean-Claude Bussard et Ingrid Brühwiler ont déjà indiqué qu'à la fin du XIX ${ }^{\mathrm{e}}$ siècle, il existait des conflits entre les promoteurs de ce que l'on pourrait considérer comme une pratique nationale, telle la gymnastique, et une pratique étrangère, comme le football. Notre objectif est dès lors d'élargir la perspective et de se demander : quelles relations ont existé entre les élites sportives sélectionnées et quelles ont été les conséquences de celles-ci pour les organisations sportives?

\section{Analyser l'Élite du SPORT SUISSE}

Les cinq sports sélectionnés dans ce projet (pour rappel : le cyclisme, le football, la gymnastique, le tir ainsi que le hornuss et la lutte suisse) ont été choisis selon deux critères qui selon nous s'induisent l'un et l'autre pour la Suisse : le nombre d'adhérents dans les associations nationales et leur popularité dans la société. Notre hypothèse - inspirée par le travail de Barbara Keys sur les organisations internationales sportives (2006) - consiste à postuler que les associations nationales des cinq sports ont joué un rôle majeur dans le développement de la pratique. En effet, ces dernières ont largement participé à les diffuser sur le territoire ; défendu les intérêts sportifs devant les acteurs politiques ainsi qu'œuvré à conférer une importance au sport dans l'esprit des citoyens suisses, que ce soit par un travail de diffusion d'information grâce à leurs organes officiels ou par l'entremise des médias de masse (presse et radio) ${ }^{5}$. Comme Christian Koller l'a déjà indiqué au sujet du football, au début du siècle dernier, plusieurs associations sont en concurrence pour administrer le jeu (2009). Or ce cas n'est pas propre au ballon rond puisqu'en cyclisme, il existe également trois associations au niveau national à la fin du XIX ${ }^{\mathrm{e}}$ siècle (Union vélocipédique suisse, 1883 ; Touring Club suisse, 1896 ; Union cycliste suisse, 1897), dont certaines entretiennent des relations concurrentielles.

Tableau 1. Principales associations nationales pour le cyclisme, le football, la gymnastique, le tir et « les jeux nationaux» (1860-1930)

\begin{tabular}{|c|c|c|c|}
\hline Nom* & Sport & Date de fondation & Siège actuel \\
\hline $\begin{array}{c}\text { Association suisse des } \\
\text { carabiniers }\end{array}$ & tir & 1824 & Berne \\
\hline $\begin{array}{c}\text { Société fédérale de } \\
\text { gymnastique }\end{array}$ & gymnastique & 1832 & Aarau \\
\hline Union vélocipédique suisse & cyclisme & 1883 & Granges \\
\hline $\begin{array}{c}\text { Association suisse de } \\
\text { football }\end{array}$ & football & 1895 & Vernier \\
\hline Touring-Club suisse & cyclotourisme & 1896 & Berthoud \\
\hline $\begin{array}{c}\text { Fédération suisse de } \\
\text { hornuss }\end{array}$ & hornuss & 1902 & \\
\hline
\end{tabular}

* nom officiel de l'association au moment de la période étudiée dans le projet

C'est pourquoi, s'il est nécessaire de bien décrire la chronologie du développement de ces organismes ainsi que leurs objectifs, nous souhaitons également examiner le lien (coopération et/ou concurrence) entre eux, ceci à l'intérieur de leurs domaines (par exemple entre les trois organes cyclistes nationaux

5 Il faut souligner que de la fin des années 1880 aux années 1930, la frontière n'est pas clairement établie entre sportifs et journalistes. Il arrive souvent que des sportifs soit eux-mêmes journalistes. C'est par exemple le cas de François Dégerine, l'un des promoteurs des sports modernes à Genève et qui est également journaliste. Il participe notamment à fonder le premier journal sportif de Suisse romande, La Suisse sportive, à la fin du XIX ${ }^{\mathrm{e}}$ siècle. 
existants à l'époque) mais aussi entre toutes ces différentes pratiques. Ces démarches aideront à cartographier le développement des associations nationales des principaux sports pratiqués en Suisse.

Pour réaliser notre étude, il s'agira de récolter de la documentation conservée dans les archives des associations nationales, qui restent pour la plupart d'entre elles largement inexplorées. En effet, hormis les archives de l'Association suisse de football, ce type de matériel n'a jusqu'ici été que très peu utilisé. Ces fonds sont majoritairement composés de communications officielles (rapports annuels du secrétaire) et des procès-verbaux des assemblées générales ou du comité exécutif. Il s'y trouve aussi souvent des dossiers spécifiques (sur l'organisation d'un concours/événement par exemple) et parfois aussi de la correspondance entre les membres ${ }^{6}$.

Afin de dépasser l'écriture d'une histoire institutionnelle qui serait essentiellement descriptive et aurait trait au fonctionnement de l'organisation et à son développement au fil du temps, nous souhaitons également placer la focale sur l'élite de ces cinq associations. Ce type de démarche s'inspire des travaux prosopographiques conduits par des chercheurs français sur les athlètes (Erard \& Bancel, 2008), les éducateurs (Al Boujjoufi \& Mierzejewski, 2007) ou les dirigeants sportifs (par exemple : Schotté, 2016). Pour le cas suisse, si des écrits scientifiques de type biographique ont déjà été publiés sur certains dirigeants (Homburg, 2007 ; Quin, 2014 ; Quin, Jaccoud \& Vonnard, 2019), l'approche proposopographique n'a pas encore été utilisée. Il s'agit dès lors de répondre à ce manque. En outre, notre ambition de s'intéresser aux élites sportives s'inscrit dans le sillage d'études historiques et sociologiques récentes menées sur les élites économiques (pour une synthèse, voir Mach et al., 2016), politiques (par exemple, études autour des députés : Pilotti, 2017) ou académiques (par exemple, des études autour des professeurs d'économie : Rossier, 2017) suisses.

D'un point de vue théorique, nous considérons ici les élites sous l'angle de la position (Genieys, 2007). En d'autres termes, nous avons sélectionné tous les dirigeants qui travaillent au sommet (soit au sein du conseil d'administration) des associations nationales. Par le biais de cette recherche, notre objectif principal est de décrire le profil et la trajectoire de ces dirigeants, puis de comprendre pourquoi ils ont investi beaucoup de temps (et parfois d'argent) dans le développement des sports. Nous soutenons que ce n'est pas seulement leur passion (sans doute sincère) pour la pratique qui explique leur action, mais aussi d'autres ambitions : sociales, économiques, voire même politiques. À cet égard, nous nous sommes inspirés d'études préliminaires biographiques collectives menées notamment par Hans-Dieter Gerber sur le développement du football à Bâle (Gerber, 2007). Dans un texte publié en 2007, ce chercheur a montré par exemple que les principaux dirigeants du football de la région de Bâle n'étaient en réalité pas seulement importants dans le domaine du sport. Il a ainsi remarqué que Paul Buser, l'un des principaux dirigeants du club Old Boys et président de l'Association suisse de football, puis des délégués de l'ASF à la Fédération internationale de football (FIFA) lors de certains congrès, tenait un rôle important dans la vie économique et politique bâloise. Dans les faits, Buser était un membre de longue date du parti radical-libéral et occupait un poste important dans l'organisme de contrôle du trafic fluvial du Rhin à Bâle (Gerber, 2007, pp. 19-20). À une époque où le football était en plein essor dans la cité rhénane, le profil international de Paul Buser a probablement aidé le ballon rond à s'implanter dans la ville, mais nous pouvons également faire l'hypothèse que le sport - grâce aux nombreux liens personnels

6 Selon Grégory Quin, la Suisse est l'un des pays où il est possible de trouver les collections de documents de première main les plus importantes dans les instances nationales du sport (2017). 
qu'il permet d'établir au travers des rencontres avec des voisins allemands notamment - a aussi pu lui servir dans son travail quotidien.

Un autre exemple qui permet d'illustrer cette situation est celui d'Adrien Thelin. Membre de l'Association suisse des carabiniers, dont il a été le président de 1893 à 1911, il était très actif dans le domaine politique au niveau local (canton de Vaud) puis national (en siégeant respectivement dans les deux différentes chambres fédérales). Impliqué dans le secteur du vin, il a également occupé un poste élevé dans l'armée et a été membre de la société étudiante Helvetia, qui a œuvré au développement de l'identité suisse. À quelle échelle et selon quelles modalités le sport a-t-il influencé sa carrière politique ? De même, a-t-il utilisé le sport comme un outil de promotion de l'identité nationale suisse à une époque où le pays était activement à la recherche d'une unité nationale (Zimmer, 2003 ; Humair, 2009) ?

Pour mieux comprendre l'action et la motivation de ces acteurs, il faudra tout d'abord identifier notre population. Pour ce faire, nous lirons les procès-verbaux des assemblées générales des cinq organes nationaux ainsi que les rapports annuels rédigés par leur secrétaire général. En effet, ces documents donnent souvent des informations précises sur les membres des associations. Dans un deuxième temps, nous allons collecter de la documentation sur les membres eux-mêmes. Dans ce domaine, nous convoquerons différents types de matériaux et d'approches. Des informations biographiques peuvent être trouvées :

- sur des sites internet (comme le Dictionnaire historique de la Suisse en ligne) ;

- dans les journaux sportifs ou généralistes, notamment lors d'interviews ou dans les nécrologies (sur ce point, la Bibliothèque nationale suisse dispose d'une base de données appelée biokat qui pourrait nous aider : https://www.helveticarchives.ch/ volltextsuche.aspx) ;
- dans les bulletins/livres sur les dirigeants publiés par les clubs/associations locaux ou les associations nationales pour des occasions spéciales (par exemple lors de leur anniversaire) ainsi que dans les livres commémoratifs parus à l'occasion de leur cinquantenaire ou centenaire.

Il sera également important de savoir si ces personnes ont obtenu des récompenses nationales (de la part de la Suisse ou de pays étranger) ou internationales et, dans l'affirmative, de trouver le dossier créé par l'association qui a décerné le prix à cette occasion. Enfin, nous devrons vérifier l'existence d'archives personnelles (archives des associations nationales, archives nationales suisses, archives des villes ou même des entreprises). Dans ce domaine, il serait également intéressant d'entrer dans des relations avec les descendants des familles et peut-être de faire des interviews.

Inspirés par les recherches de collègues suisses ayant participé aux études de l'Observatoire suisse des élites (OBELIS $)^{7}$, nous créerons une base de données FileMaker contenant des informations sur toutes les élites sélectionnées dans le cadre de notre projet. Cette base de données nous aidera d'abord à centraliser les informations et à les analyser (éventuellement dans le but de procéder ensuite à une analyse de réseau). De plus, cela pourrait aussi être l'occasion de faire mieux connaitre notre recherche. En effet, à la fin du projet, l'idée est de donner au public l'occasion de consulter une partie de ce résultat (peut-être par le biais d'un site Web ou directement dans la base de données OBELIS).

Notre équipe de recherche est composée de quatre personnes qui travaillent toutes à l'Institut des sciences du sport de l'Université de Lausanne (ISSUL) :

- Grégory Quin, maître d'enseignement et de recherche, est le requérant principal du projet. Il va particulièrement s'intéresser au cas du tir et des « jeux nationaux ». 
- Philippe Vonnard, chercheur FNS senior, est le coordinateur scientifique du projet. Il va essentiellement s'intéresser aux cas du cyclisme et du football.

- Gil Mayencourt, doctorant FNS, débute une thèse (sous la direction de Grégory Quin) sur les premiers développements de la gymnastique et du cyclisme en Suisse tout en portant une attention particulière à leur rôle dans la construction de l'identité suisse.

- Hans-Dieter Gerber, collaborateur scientifique, apportera une perspective de recherche plus locale en se consacrant au développement du football dans la région de Bâle.

L'équipe est complétée par Sébastien Cala. Assistant-diplômé à l'ISSUL, il prépare actuellement une thèse (sous la supervision de Grégory Quin et de Claude Hauser, professeur d'histoire contemporaine à l'Université de Fribourg) autour de la relation entre le ski et le tourisme dans une perspective comparée entre plusieurs régions alpines de Suisse.

\section{ConcLusion}

Pour terminer, un bref point sur les résultats attendus du projet et sur ses perspectives. En termes de diffusion de la recherche, nous prévoyons de participer chaque année à des colloques (congrès d'histoire du sport, mais aussi d'histoire mondiale et contemporaine) et d'organiser deux ateliers de recherche par année avec d'autres collègues historiens suisses. Au terme du projet, l'objectif est d'organiser à l'Université de Lausanne un colloque international sur les enjeux de la « nationalisation " du sport, en mettant l'accent sur différents aspects : le transfert de la culture sportive d'un pays à un autre ; le développement d'associations sportives nationales et les motivations des grands leaders sportifs nationaux.

En ce qui concerne l'aspect " publications », les thèses de Sébastien Cala et de Gil
Mayencourt constitueront l'un des principaux résultats du projet. En outre, sur la base de ce qu'ont pu réaliser des collègues à la fin de projets de recherche analogues (par exemple : Humair et al., 2014), nous souhaitons rédiger un ouvrage de synthèse sur les premiers développements institutionnels des sports en Suisse. Celui-ci sera publié dans la collection « Sport et sciences sociales " hébergée aux Presses universitaires suisses (Alphil, Neuchâtel). Notre objectif est également de viser quelques publications dans des bonnes revues scientifiques sur l'histoire du sport et/ou l'histoire générale.

Ce projet de recherche doit permettre de mieux comprendre le développement des principaux sports en Suisse et la place qu'ils ont progressivement pris dans la société helvétique. En outre, nous souhaitons poser de solides jalons en vue de développer de futurs projets de recherches d'envergure sur l'histoire des sports en Suisse. De plus, ce projet permettra d'engager une discussion avec des collègues étrangers qui ont étudié le développement des sports dans leur pays, démarche qui n'a jusqu'ici pas véritablement été possible en Suisse en raison de la relative faiblesse de l'historiographie en la matière.

Enfin, notre travail offrira un aperçu des premiers membres de l'élite sportive suisse. En nous concentrant sur leurs motivations, nous comprendrons mieux pourquoi ils se sont engagés dans l'administration des sports, mais aussi comment ces activités, au premier abord futiles, ont pu dans les faits contribuer à optimiser leur carrière économique et politique. Ce dernier point donnera aux historiens du sport la possibilité de créer un dialogue avec des collègues suisses et étrangers travaillant dans les domaines de l'élite culturelle, économique, politique et scientifique. À ce titre, et last but not least, ce projet contribuera - et nous l'espérons sincèrement - à faire reconnaître le sport comme un domaine de recherche désormais légitime au sein d'une historiographie suisse elle-même en plein renouvellement (Eichenberger et al., 2017). 


\section{BiBLIOGRAPHIE}

Al Boujjoufi, T. \& Mierzejewski, S. (2007). Le recrutement socio-professionnel du personnel enseignant des Instituts Régionaux d'Éducation physique (1927-1939). Staps, 75(1), 9-24.

Anderson, B. (1996). Limaginaire national. Paris : La Découverte.

Arnaud, P. (1998). Les athlètes de la République. Gymnastique, sport et idéologie républicaine 1870-1914. Paris : L'Harmattan.

Barton, S. (2008). Healthy Living in the Alps : sanatoria and the origins of winter sports tourism in Switzerland, 1860-1914. Manchester : Manchester University Press.

Berthoud, J., Quin, G. \& Vonnard, P. (2016). Le football suisse. Des pionniers aux professionnels. Lausanne : Presses polytechniques et universitaires romandes.

Berthoud, J., Quin, G. \& Vonnard, P. (2018). Le retour du professionnalisme dans le football suisse d'élite (1947-1988). In T. Busset, B. Fincoeur \& R. Besson (dir.), En marge des grands : le football en Belgique et en Suisse (pp. 123-145). Berne : Peter Lang.

Brändle, F. \& Koller, C. (2014). 4 zu 2. Die Goldene Zeit des Schweizer Fussballs 1918-1939. Göttingen : Verlag die Werkstatt.

Brühwiler, I. (2017). In-between " Swedish Gymnastics " and "Deustche Turn-Kunst » : Educating " National " Citizens through Physical Education in Switzerland in the Last Decades of the Nineteenth Century. Nordic Journal of Educational History, 4(2), 71-84.

Burgener, L. (1952). La Confédération suisse et l'éducation physique de la jeunesse. Thèse de doctorat, Université de Genève.

Bussard, J.-C. (2007). L'éducation physique suisse en quête d'identité (1800-1930). Paris : L'Harmattan.

Busset, T. (2001). De la sociabilité mondaine à la compétition : les débuts du hockey sur glace en Suisse. In C. Jaccoud \& T. Busset (dir.), Sports en formes. Acteurs, contextes et dynamiques d'institutionnalisation (pp. 127-136). Lausanne : Antipodes.

Busset, T. (2016). La diffusion du ski en Suisse jusqu'à l'entre-deux-guerres. Traverse. Revue d'histoire, 23(1), 25-36.

Busset, T. \& Marcacci, M. (dir.) (2006). Pour une histoire des sports d'hiver. Zur Geschichte des Wintersports. Actes du colloque de Lugano, 20 et 21 février 2004. Neuchâtel : CIES.
Busset, T., Jucker, M. \& Koller, C. (dir). Histoire du sport en Suisse. État des lieux et perspectives. Neuchâtel : CIES.

Cala, S. (2019). Ski et tourisme dans la Vallée de Joux, à la croisée des intérêts sportifs et touristiques (18991939). Entreprises et histoire, 93(4), 62-74.

Cronin, M., Duncan, M. \& Rouse, P. (2009). The GAA. A people's history. Cork : Collins Press.

Czaka, V. (2017). Histoire sociale de l'éducation physique en Suisse romande (milieu XIXe siècle-début XX $X^{e}$ siècle). Thèse de doctorat, Université de Genève.

Eichenberger, L. (1994). Die Eidgenossische Sportschule Maggligen. Macolin : Éditions EFSM.

Eichenberger, P. et al. (2017). Beyond Switzerland. Reframing the Swiss Historical Narrative in Light of Transnational History. Traverse. Revue d'histoire, 27(1), 137-152.

Eisenberg, C. (1999). "English sports » und deutsche Bürger. Eine Gesellschaftsgeschichte 1800-1939. Paderborn : F. Schöningh.

Erard, C. \& Bancel, N. (2008). Prosopographical analysis of sports elites : Overview and evalutation of a seminal study. The International Journal of the History of Sport, 24(1), 67-79.

Favre, C. (2004). La Suisse face aux Jeux olympiques de Berlin 1936. Fribourg : Aux sources du temps présent.

Favre, F. \& Vonnard, P. (2015). Un tourisme sportif? Le rôle des hôteliers dans l'apparition des sports dans la région de Montreux (1880-1914). Revue historique vaudoise, 123, 219-233.

Gaboriau, P. (1991). Les trois âges du vélo en France. 20 E 21. Revue d'histoire, 29(1), 17-34.

Genieys, W. (2007). Nouveaux regards sur les élites du politique. Revue française de science politique, 56(1), 121-147.

Gerber, H.-D. (2001). Die Gründerzeit des FC Basel von 1893 bis 1914. Basel : Friedrich Reinhardt Verlag.

Gerber, H.-D. (2007). Fussball in Basel von den Anfängen bis zum Zweiten Weltkrieg. Basler Zeitschrift für Geschichte und Altertumskunde, 107, 9-33.

Gigase, M., Jucker, M. \& Koller, C (2016). Masse, Märkte und Macht in der Geschichte des Sports : Editorial = Masse, marchés et pouvoir dans l'histoire du sport : Éditorial. Traverse. Revue d'histoire, 23(1), 8-24.

Gillabert, M. (2013). Dans les coulisses de la diplomatie culturelle suisse. Objectifs, réseaux et réalisations (19381984). Neuchâtel : Alphil.

Giuliani, M. (2001). "Starke Jugend - Freies Volk". Bundesstaatliche Körpererziehung und Gesellschaftlische 
Funktion von Sport in der Schwiez (1918-1947). Berne : Peter Lang.

Gogniat, J. (2018). L'éducation des corps dans les pensionnats et l'émergence du sport en Suisse au tournant du XIX ${ }^{\mathrm{e}}$ siècle, In M. Aceti, C. Jaccoud $\&$ L. Tissot (dir.), Faire corps. Temps, lieux et gens (pp. 45-58). Neuchâtel : Alphil.

Guggisberg, P. (2009). 75 ans Swiss Football League Ligue nationale ASF. Berne : Muri.

Heller, G. (1988). «Tiens-toi droit! ». L'enfant à l'école au $X I X^{e}$ siècle : espace, morale et santé. L'exemple vaudois. Lausanne : Éditions d'en Bas.

Hobsbawn, Eric (1990). Nations et nationalisme depuis 1780. Paris : Gallimard.

Holt, R. (1981). Sport and Society in Modern France. London: Macmillan.

Homburg, H. (2007). Ernst Thommen, die Schweiz und der Weltfussball, 1946-1962. Basler Zeitschrift für Geschichte und Altertumskunde, 107, 69-102.

Humair, C. (2009). 1848. Naissance de la Suisse moderne. Lausanne : Antipodes.

Humair, C. et al. (2014). Système touristique et culture technique dans l'arc lémanique. Analyse d'une success-story et de ses effets sur l'économie régionale (1852-1914). Neuchâtel : Alphil.

Jost, H.-U. (2001). Critique historique du consensus helvétique : si le légendaire Guillaume Tell et le mythe du serment du Grütli ne nourrissent plus guère l'imaginaire de la jeune génération, la Suisse comme pays modèle du consensus est un cliché qui se porte bien. Traverse. Revue d'histoire, 8(1), 57-79.

Keys, B. (2006). Globalizing Sport. National Rivalry and International Community in the 1930s. Harvard : Harvard University Press.

Koller, C. (2009). Associations de football concurrentes pendant la première moitié du $20^{\mathrm{e}}$ siècle. In N. Bancel, T. David, F. Ohl (dir.), Le football en Suisse. Enjeux sociaux et symboliques d'un spectacle universel (pp. 31-46). Neuchâtel : Éditions CIES.

Koller, C. (2010). Football negotiating the placement of Switzerland within Europe. Soccer E Society, 11(6), 748-760.

Koller, C. (2012). Sport, Urbanity and Communal Socialism: The Case of « Red Zurich » (1928-1949). The International Journal of the History of Sport, 29(14), 2013-2029.

Koller, C. (2017). Sport transfer over the channel : elitist migration and the advent of football and ice hockey in Switzerland. Sport in Society, 20(10), 1390-1404.
Lanfranchi, P. (1998). Football et modernité. La Suisse et la pénétration du football sur le continent. Traverse. Revue d'histoire, 3, 76-87.

Lemercier, C. \& Picard, E. (2011). Quelle approche prosopographique? In P. Nabonnand \& L. Rollet (dir.), Les uns et les autres... Biographies et prosopographies en histoire des sciences (pp. 605-630). Nancy : Presses universitaires de Nancy.

Mach, A. et al. (2016). Les élites économiques suisses au $\mathrm{XX}^{\mathrm{e}}$ siècle. Neuchâtel : Alphil.

Pécout, G. (1990). Les Sociétés de tir dans l'Italie unifiée de la seconde moitié du XIX siècle. Mélanges de l'École française de Rome. Italie et Méditerranée, 102(2), 533-676.

Pfister, G. (2003). Cultural Confrontations : German Turnen, Swedish Gymnastics and English Sport - European Diversity in Physical Activities from a Historical Perspective. Sport in Society : Culture, Sport, Society, 6(1), 61-91.

Philipona Romanens, A. (1999). Le développement du ski dans le canton de Fribourg (1930-1960). Fribourg : Aux sources du temps présent.

Pieth, F. (1979). Sport in der Schweiz. Sein Weg in die Gegenwart. Olten : Walter Verlag.

Pilotti, A. (2017). Entre démocratisation et professionnalisation : le Parlement suisse et ses membres de 1910 à 2016. Zuurich : Seismo Verlag.

Quin, G. (2010). La Suisse face à la Grande Allemagne (1933-1942). Éléments pour une histoire du football helvétique. In M. Attali (dir.), Sports et médias, 19-20 siècles (pp. 761-770). Biarritz : Atlantica.

Quin, G. \& Vonnard, P. (2011). «Par-delà le Gothard ». Les matches internationaux Italie-Suisse et la consolidation des champs footballistiques italien et suisse dans l'entre-deux-guerres. Diacronie. Studi di Storia Contemporanea, 5, 1-14.

Quin, G. (2014). Promoteur et dirigeant «sportif » : Francis Messerli (1888-1975) pionnier de l'organisation du sport helvétique. In E. Bayle (dir.), Les grands dirigeants et managers du sport. Trajectoires, pratiques et héritages (pp. 45-57). Bruxelles : De Boeck.

Quin, G \& Vonnard, P. (2015). Switzerland - a Stronghold in European Football, 1930-1954? Sport in History, 35(4), 531-549.

Quin, G. (2016). L'odyssée du sport universitaire lausannois. Entre compétition et sport-santé. Paris : Glyphe.

Quin, G. (2017). Writing Swiss Sport History: A Quest for Original Archives. The International Journal of the History of Sport, 34(5-6), 432-436.

Quin, G. (2017). De la cure d'air à l'or blanc. Une Interassociation Suisse pour le ski face aux enjeux 
de l'essor du ski en Suisse (années 1920-années 1960). Histoire des Alpes, 22, 135-155.

Quin, G., Vonnard, P. \& Jaccoud, C. (dir). (2019). Des hommes et des réseaux. Participation et contribution de la Suisse à l'internationalisation du sport. Neuchâtel : Alphil.

Rossier, T. (2017). Affirmation et transformations des sciences économiques en Suisse au XXe siècle. Thèse de doctorat, Université de Lausanne.

Schotté, M. (2016). Monter en première division. Trajectoires de notabilisation des présidents de clubs de football professionnel (1960-1999). Politix, 114(2), 99-120.

Tissot, L. (2000). Naissance d'une industrie touristique. Les Anglais et la Suisse au XIX siècle. Lausanne : Payot.

Tissot, L. (2018). Une histoire économique du football suisse au $\mathrm{XX}^{\mathrm{e}}$ siècle est-elle possible et utile? In T. Busset, B. Fincoeur \& R. Besson (dir.), En marge des grands : le football en Belgique et en Suisse (pp. 99-122). Berne : Peter Lang.

Tissot, L. \& Schneider, G. (2018). Hôtel et sport : quelles relations ? L'exemple de la Suisse alpine (XIX ${ }^{\mathrm{e}}$ siècle-1954). Entreprises et histoire, 93(8), 12-26.

Tissot, L., Quin, G. \& Vonnard, P. (2018). Un tourisme sportif? Les entreprises touristiques et le développement des sports en France et en Suisse (18501950). Entreprises et histoire, 93(8), 5-11.

Tonnerre, Q. \& Quin, G. (2017), « Les Suisses n'iront pas à Melbourne pour n'avoir pas trouvé d'avion convenable ». Histoire d'une non-participation olympique (1948-1956). Revue suisse d'histoire, 67(3), 343-360.

Tonnerre, Q. (2019). «Une question de prestige dans le domaine international de l'industrie horlogère ".
Diplomatie suisse et chronométrage sportif (19641970). Relations internationales, 177(1), 129-144.

Villiger, C. (2017). Usages de la violence en politique (19502000). Lausanne : Antipodes.

Vonnard, P. \& Quin, G. (2012). Éléments pour une histoire de la mise en place du professionnalisme dans le football suisse durant l'entre-deux-guerres : processus, résistances et ambiguïtés. Revue suisse d'histoire, 62(1), 70-85.

Vonnard, P. (2019). Institutionnaliser l'histoire du sport en Suisse. Enjeux de la création de l'Association suisse d'histoire du sport (ASHS). Traverse. Revue d'histoire, 28(1), 137-146.

Vonnard, P. \& Quin, G. (2019). Les premiers temps de l'institutionnalisation des sports modernes en Suisse. L'exemple du football des années 1870 aux années 1910. In T. Busset, M. Jucker \& C. Koller (dir), Histoire du sport en Suisse. État des lieux et perspectives (pp. 55-78). Neuchâtel : CIES.

Vonnard, P. \& Quin, G. (2019). Promouvoir l'internationalisation du football : les dirigeants suisses et la Fédération internationale de Football Association (FIFA) (1904-1954). Storia dello sport. Rivista di studi contemporanei, 1. Publié en ligne : https://storia-sport.it/index.php/sp/issue/view/1.

Walter, F. (2004). Les figures paysagères de la nation : territoire et paysage en Europe $\left(16^{-}-20^{\circ}\right.$ siècle). Paris : Éditions de l'École des hautes études en sciences sociales.

Wille, F. (2003). Le Tour de France : un modèle médiatique. Villeneuve-d'Ascq : Presses universitaires du Septentrion.

Zimmer, O. (2003). A Contested Nation : History, Memory and Nationalism in Switzerland 1761-1891. New York: Cambridge University Press.

\section{Resumen :La Fábrica Nacional de Deportes. Estudiar el desarrollo de la elite del deporte suizo (1860-1930}

En Suiza, como en otros lugares, los efectos y las resonancias de las actividades deportivas afectan las esferas económica, política, higiénica o cultural, y la institucionalización del deporte es un proceso que permite comprender singularmente la dinámica estructuradora de nuestra modernidad. Teniendo en cuenta esta situación y para comprender el importante lugar que ocupa el deporte en la sociedad suiza, el proyecto titulado "The National Sports Factory", financiado para el período 2019-2023 por la Swiss National Science Foundation (FNS): propone centrarse en la génesis y los primeros desarrollos del deporte en Suiza desde 1860 hasta 1930. Este proceso establece los principales deportes en la sociedad suiza relacinado de manera más general el desarrollo político, económico y cultural del propio país en la cual la estructura estatal moderna se remonta a 1848. La investigación se centra en el desarrollo de cinco deportes (en orden alfabético): ciclismo, gimnasia, fútbol, tiro y lo que generalmente se llama los "Juegos Nacionales" constituidos de hornuss y lucha libre suiza. 
En el marco de este artículo, se trata de volver brevemente a los objetivos de este proyecto, para luego desarrollar un aspecto central: una búsqueda exhaustiva en torno a las élites del deporte suizo.

Palabras clave : Historia, deporte, Suiza, organización, elites.

\section{Zusammenfassung: "Die Fabrik des nationalen Sports". Untersuchung der Entstehung einer Schweizer Sportelite (1860-1930)}

In der Schweiz wie anderswo beeinflussen die Auswirkungen und Resonanzen von sportlichen Aktivitäten den wirtschaftlichen, politischen, hygienischen oder kulturellen Bereich, und die Institutionalisierung des Sports ist ein Prozess, der es ermöglicht, die Strukturdynamik unserer Moderne auf einzigartige Weise zu verstehen.

Angesichts dieser Situation und um die wichtige Rolle des Sports in der Schweizer Gesellschaft zu verstehen, schlägt das vom Schweizerischen Nationalfonds (SNF) für die Periode 2019-2023 finanzierte Projekt "La Fabrique des sports nationaux" vor, sich auf die Entstehung und frühe Entwicklung des Sports in der Schweiz von 1860 bis 1930 zu konzentrieren. Dieser Prozess der Etablierung der Hauptsportarten in der Schweizer Gesellschaft folgt im Allgemeinen der politischen, wirtschaftlichen und kulturellen Entwicklung des Landes selbst, dessen moderne Staatsstruktur bis ins Jahr 1848 zurückreicht. Im Mittelpunkt der Forschung steht die Entwicklung von fünf Sportarten (in alphabetischer Reihenfolge): Radfahren, Gymnastik, Fußball, Schießen und die "Nationalsportarten" Hornussen und Schwingen (Schweizer Ringen).

In diesem Artikel gehen wir kurz auf die Ziele dieses Projekts ein und entwickeln daraus einen zentralen Aspekt: die umfangreiche Forschung rund um die Eliten des Schweizer Sports.

Schlagwörter: Geschichte; Sport, Schweiz, Organisation, Eliten

\section{"La Fabrique des sports nationaux 》. Studiare l'emergere di un'élite dello sport Svizzero (1860-1930)}

In Svizzera, come altrove, gli effetti e le risonanze delle attività sportive toccano nello stesso tempo le sfere economiche, politiche, igieniche o cultuali, e l'istituzionalizzazione dello sport è un processo che permette di comprendere singolarmente delle dinamiche strutturanti della nostea modernità.

Tenendo conto di questa situazione e con lo scopo di comprendere il posto importante preso dallo sport nella società svizzera, il progetto intitolato «La Fabrique des sports nationaux » - finanziato per il periodo 2019-2023 dal Fond national suisse pour la recherche scientifique (FNS) - propone di concentrarsi sulla genesi e i primi sviluppi degli sport in Svizzera dal 1960 al 1930. Questo processo di istituzione dei principali sport nella società elvetica segue più generalmente lo sviluppo politico, economico e culturale del paese stesso la cui struttura statale moderna data dal 1848. La ricerca porta sullo sviluppo di cinque sport (per ordine alfabetico) : il ciclismo, la ginnastica, il football, il tiro, e ciò che si chiamano generalmente i " Giochi nazionali » costituiti dall'hornuss e la lotta svizzera.

Nel quadro di questo articolo si tratta di ritornare brevemente sugli obiettivi di questo progetto, poi di sviluppare un aspetto centrale di quest'ultimo : la ricerca estensiva impegnata attorno alle élite dello sport svizzero.

Parole chiave: élite, organizzazioni, sport, storia, Svizzera 